\title{
Management of nicotinamide $N$-methyltransferase overexpression: inhibit the enzyme or reduce nicotinamide intake?
}

\author{
Shi-Sheng Zhou ${ }^{1} \cdot \mathrm{Da} \mathrm{Li}^{2} \cdot$ Yiming Zhou ${ }^{3}$
}

Received: 27 May 2015 / Accepted: 8 June 2015 / Published online: 30 June 2015

(C) Springer-Verlag Berlin Heidelberg 2015

Keywords 1-Methylnicotinamide $\cdot$ Cancer $\cdot$ Insulin resistance $\cdot \mathrm{NAD}^{+} \cdot$ Nicotinamide $\cdot$ Nicotinamide $N$-methyltransferase $\cdot$ Nicotinic acid $\cdot$ Obesity $\cdot$ Type 2 diabetes

\section{Abbreviations \\ MNA 1-Methylnicotinamide \\ NNMT Nicotinamide $N$-methyltransferase}

To the Editor: In a recent issue of this journal, Kannt et al reported that the expression of the gene encoding nicotinamide $N$-methyltransferase (NNMT) in adipose tissue is increased in patients with insulin resistance and type 2 diabetes, and that the plasma level of 1-methylnicotinamide (MNA) is significantly correlated with NNMT expression [1]. Their findings expand the current understanding of the role of disturbed nicotinamide metabolism in insulin resistance-related diseases.

The authors suggest that inhibiting NNMT may provide a novel therapeutic approach for insulin resistance. We believe that it would be inadvisable to use this approach as it does not

Shi-Sheng Zhou

zhouss@ymail.com

1 Institute of Basic Medical Sciences, Medical College, Dalian University, No.10 Xuefu Avenue, Dalian Economic and Technological Development Zone, Dalian 116622, People's Republic of China

2 Department of Obstetrics and Gynecology, Shengjing Hospital of China Medical University, Shenyang, People's Republic of China

3 Renal Division, Department of Medicine, Brigham and Women's Hospital, Harvard Institutes of Medicine, Harvard Medical School, Boston, MA, USA take into account the causal role of excess nicotinamide intake in the development of insulin resistance and type 2 diabetes. Therefore, we would like to offer some comments on this article.

As shown in Fig. 1, nicotinamide is degraded to MNA by NNMT. The major metabolites of nicotinamide in human urine are MNA and its oxidation product $N^{1}$-methyl-2pyridone-5-carboxamide. This indicates that NNMT is a key enzyme responsible for the clearance of excess nicotinamide. It has been demonstrated in animal studies that nicotinamide can induce Nnmt expression [2]. Thus, the association of increased NNMT expression with high plasma MNA levels should be a consequence of excess nicotinamide intake, rather than a primary cause of insulin resistance. In fact, over the past several decades, increased prevalence of obesity and type 2 diabetes is closely correlated with increased nicotinamide intake, due to nicotinamide fortification and a high intake of animal-derived foods [3-5]. High fasting nicotinamide levels have been observed in hypertension, a disorder closely related to insulin resistance [6]. Therefore, to explore the cause of increased expression of NNMT and high levels of serum MNA, nicotinamide intake and blood nicotinamide status should also be taken into consideration.

Bariatric surgery is known to significantly reduce food intake, which will undoubtedly decrease nicotinamide intake. Thus, the most likely explanation for the reduction in plasma MNA and NNMT expression in adipose tissue after surgery is due to decreased nicotinamide intake. In this regard, we suggest that, before coming to a conclusion about the role of NNMT and MNA in insulin resistance and type 2 diabetes, the authors should consider the effect of bariatric surgery on the total daily intake of nicotinamide.

It is known that nicotinamide is hardly excreted in the urine because of reabsorption by renal tubules, but it can be effectively excreted in sweat [5]. We have observed that there is a 


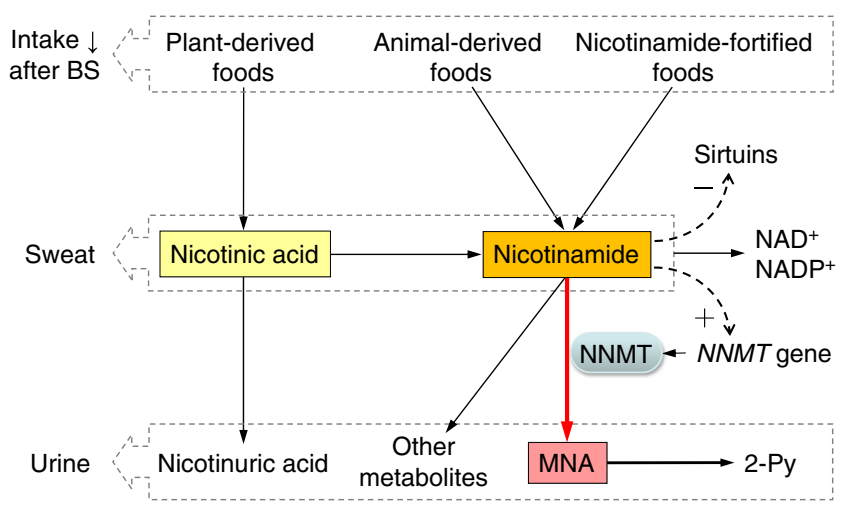

Fig. 1 The sources and fates of nicotinamide. BS, bariatric surgery; 2-Py, $N^{1}$-methyl-2-pyridone-5-carboxamide. -, inhibiting effect; +, inducing effect

more than fivefold increase in sweat nicotinamide with no change in MNA levels after an oral nicotinamide load $(100 \mathrm{mg})$ [7]. Intensive exercise is accompanied by increased sweat excretion, so the finding that endurance training can decrease $N N M T$ expression in individuals with insulin resistance or type 2 diabetes may involve increased sweat excretion of nicotinamide. Therefore, it is necessary to examine the effect of exercise on blood nicotinamide status when exploring the effects of exercise on NNMT expression, plasma MNA and insulin resistance.

Nicotinamide is a precursor in the synthesis of $\mathrm{NAD}^{+}$and $\mathrm{NADP}^{+}$, two coenzymes that play roles in numerous biological processes related to redox. A high nicotinamide intake may enhance these biological processes by affecting the synthesis of $\mathrm{NAD}^{+}$and $\mathrm{NADP}^{+}$, and subsequently the activity of $\mathrm{NAD}^{+}$- and $\mathrm{NADP}^{+}$-dependent enzymes. In theory, inhibition of NNMT may have similar effects to excess nicotinamide, which is known to cause insulin resistance, as the authors mention in the introduction of their paper [1]. Moreover, nicotinamide can inhibit sirtuins, which are NAD-dependent protein deacetylases known to have protective effects against agerelated diseases, including diabetes and cancer [8]. As noted by the authors, cancer is associated with high NNMT expression [1]. This suggests the possibility that excess nicotinamide may contribute to cancer. From this point of view, inhibiting NNMT may lead to unpredictable consequences.
Taken together, we argue that what the authors observed in their study is actually a reflection of changes in nicotinamide intake and excretion. Given that (1) high nicotinamide intake is very common in developed countries, and (2) excess nicotinamide is known to cause oxidative stress and insulin resistance $[3,5]$, we think it wise to reduce nicotinamide intake and increase its excretion rather than to inhibit NNMT in the management of diseases related to $N N M T$ overexpression.

Funding This work was supported by the National Natural Science Foundations of China (Grant nos: 31140036 to SSZ and 81402130 to DL).

Duality of interest The authors declare that there is no duality of interest associated with this manuscript.

Contribution statement All authors were responsible for drafting the article, revising it critically for important intellectual content, and approving the version to be published.

\section{References}

1. Kannt A, Pfenninger A, Teichert L et al (2015) Association of nicotinamide- $N$-methyltransferase mRNA expression in human adipose tissue and the plasma concentration of its product, 1methylnicotinamide, with insulin resistance. Diabetologia 58:799-808

2. Tian YJ, Luo N, Chen NN et al (2014) Maternal nicotinamide supplementation causes global DNA hypomethylation, uracil hypoincorporation and gene expression changes in fetal rats. Br J Nutr 111:1594-1601

3. Li D, Sun WP, Zhou YM et al (2010) Chronic niacin overload may be involved in the increased prevalence of obesity in US children. World J Gastroenterol 16:2378-2387

4. Zhou SS, Li D, Zhou YM, Sun WP, Liu QG (2010) B-vitamin consumption and the prevalence of diabetes and obesity among the US adults: population based ecological study. BMC Public Health 10:746

5. Zhou SS, Zhou Y (2014) Excess vitamin intake: an unrecognized risk factor for obesity. World J Diabetes 5:1-13

6. Sun WP, Li D, Lun YZ et al (2012) Excess nicotinamide inhibits methylation-mediated degradation of catecholamines in normotensives and hypertensives. Hypertens Res 35:180-185

7. Zhou SS, Li D, Sun WP et al (2009) Nicotinamide overload may play a role in the development of type 2 diabetes. World J Gastroenterol 15:5674-5684

8. Donmez G, Outeiro TF (2013) SIRT1 and SIRT2: emerging targets in neurodegeneration. EMBO Mol Med 5:344-352 